\title{
Koszul duality in algebraic topology
}

\author{
Dev P. Sinha
}

Received: 18 October 2011 / Accepted: 20 March 2012 / Published online: 16 May 2012

(C) Tbilisi Centre for Mathematical Sciences 2012

\begin{abstract}
The most prevalent examples of Koszul duality of operads are the selfduality of the associative operad and the duality between the Lie and commutative operads. At the level of algebras and coalgebras, the former duality was first noticed as such by Moore, as announced in his ICM talk at Nice (Moore in Actes du Congrès International des Mathématiciens, Tome 1. Gauthier-Villars, Paris, pp. 335-339, 1971). This particular duality has typically been called Moore duality, and some prefer to call the general phenomenon Koszul-Moore duality. The second duality at the level of algebras was realized in the seminal work of Quillen on rational homotopy theory (Quillen in Ann Math 90(2):205-295, 1969). Our aim in these notes based on our talk at the Luminy workshop on Operads in 2009 is to try to provide some historical, topological context for these two classical algebraic dualities. We first review the original cobar and bar constructions used to study loop spaces and classifying spaces, emphasizing the less-familiar geometry of the cobar construction. Then, after some elementary topology, we state duality between bar and cobar complexes in that setting. Before explaining Quillen's work, we also share some other ideas-calculations of Cartan-Serre and Milnor-Moore and philosophy of Eckmann-Hilton-which may have influenced him. After stating Quillen's duality, we share some recent work which relates these constructions to geometry through Hopf invariants and in particular linking phenomena.
\end{abstract}

Keywords Koszul-Moore duality · Bar and cobar constructions · Operads . Hopf invariants

Communicated by Jim Stasheff.

D. P. Sinha $(\varangle)$

Department of Mathematics, University of Oregon,

Eugene, OR 97403, USA

e-mail:dps@math.uoregon.edu 


\section{Bar and cobar constructions}

\section{$1.1 \Omega X$ and the cobar construction}

Studying mapping spaces is one of the central tasks of topology, and loop spaces are the simplest and most fundamental examples (unless one counts maps from finite sets, which yield products). We require a model for loops where the loop sum is associative exactly, not up to homotopy. For us $\Omega X$ denotes the Moore loop space which consists of pairs $f: \mathbb{R} \rightarrow X$ and a "curfew" $a>0$ such that $f(x)$ is the basepoint if $x \leq 0$ or if $x \geq a$. Loop sum adds these curfews, which makes multiplication associative.

The cobar construction of Adams and Hilton [2] was informed by the almost concurrent work of James [10] who studied $\Omega \Sigma X$, the loop space on the reduced suspension of $X$, namely $\Sigma X=X \times \mathbb{I} /(X \times 0 \cup * \times \mathbb{I} \cup 1 \times X)$. There is a canonical inclusion of $J: X \hookrightarrow \Omega \Sigma X$ sending $x$ to $J(x)(t)$, the path which sends $t$ to the image in $\Sigma X$ of $(x, t)$. Because $\Omega \Sigma X$ is a topological monoid, this map extends to a map from the free moinoid (with unit) on $X$ to $\Omega \Sigma X$ which we call the James map $\hat{J}$. For example, the formal product $y * x * z$ goes to a loop with coordinates $(x, t)$ for $t \in[0,1]$ then $(y, t-1)$ for $t \in[1,2]$, then $(z, t-2)$ for $t \in[2,3]$ - see Fig. 1 below.

Theorem 1.1 (James [10]) The James map $\hat{J}$ from the free monoid on $X$ to $\Omega \Sigma X$ is a homotopy equivalence.

Recall that the homology of any space with an associative multiplication, or even a homotopy associative multiplication, is an associative algebra.

Corollary 1.2 The homology of $\Omega \Sigma X$ with field coefficient is isomorphic as an algebra to the tensor (that is, free associative) algebra on the homology of $X$.

Fig. 1 An illustration of $\hat{J}$ of $y * x * z$ (traversing the path through $y$ first, etc)

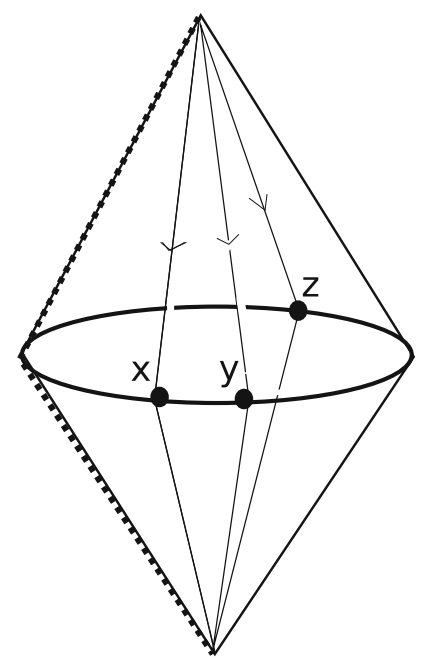


Fig. 2 One possible choice for $\gamma_{2}$
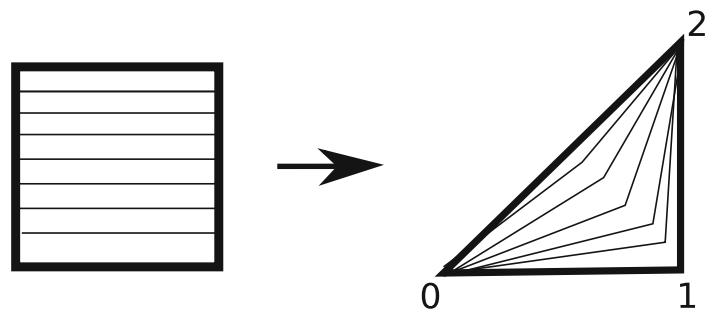

Exercise 1 Explicitly define the free topological monoid on a (well-based) topological space X. Show that its homology with field coefficients is isomorphic to the tensor algebra on the homology of X. [Hint: make heavy use of the Künneth theorem.]

Interestingly, the corollary is typically proven in the course of proof of the theorem. Details in a well-digested form are in Sect. 4.J in Hatcher's textbook [9] or the survey paper of Carlsson and Milgram [4], whose treatment of the Adams-Hilton construction heavily influences our treatment below. There are however more geometric proofs which build on the fact that the space of paths in the cone on $X$ with endpoints in the image of $X$ is homotopy equivalent to $X \times X$ through the projection onto the endpoints.

For the Adams-Hilton construction, we start with a simply-connected simplicial complex $\hat{X}$ and then contract the union of the 1-skeleton along with enough of the two-skeleton so that the quotient map $\hat{X} \rightarrow X$ is a homotopy equivalence. Then $X$ is a CW-complex, and its cellular chains are a quotient of the simplicial chains on $\hat{X}$. By abuse of notation, we denote these cellular chains by $C_{*}^{\Delta}(X)$.

Next, consider the cubical singular chain complex of the loop space $C_{*}^{\square}(\Omega X)$, which is an associative differential graded algebra. On generators, the product of $\sigma_{1}: \mathbb{I}^{n} \rightarrow$ $\Omega X$ and $\sigma_{2}: \mathbb{I}^{m} \rightarrow \Omega X$ is the composite $\mathbb{I}^{n+m} \cong \mathbb{I}^{n} \times \mathbb{I}^{m} \stackrel{\sigma_{1} \times \sigma_{2}}{\rightarrow} \Omega X \times \Omega X \rightarrow \Omega X$.

The Adams-Hilton construction defines a map of associative algebras from the free associative algebra on $C_{*}^{\Delta}(X)$ to $C_{*}^{\square}(\Omega X)$. The first key observation is that any choice of map $\gamma_{n}: \mathbb{I}^{n} \rightarrow \Delta^{n}$ defines a map $A H_{\gamma_{n}}: C_{n}^{\Delta}(X) \rightarrow C_{n-1}^{\square}(\Omega X)$. Let $\chi_{\sigma}$ denote the characteristic map $\Delta^{n} \rightarrow X$ of a simplex $\sigma$ of $X$. Then $A H_{\gamma_{n}}(\sigma)$ is basically given by the composite $\gamma_{n} \circ \chi_{\sigma}: \mathbb{I}^{n} \rightarrow X$. From this composite we by adjointness (choosing say the last coordinate as the loop coordinate) produce a map $\mathbb{I}^{n-1} \rightarrow \operatorname{Map}(\mathbb{I}, X)$, which then is identified with a generator of $C_{n-1}^{\square}(\Omega X)$ through viewing $\operatorname{Map}(\mathbb{I}, X)$ as Moore loops with curfew one.

The game is to define $\gamma_{n}$ appropriately so that we can calculate boundaries, and more importantly so that the Adams-Hilton map yields a quasi-isomorphism. By abuse, we suppress $\gamma_{n}$ from notation and write $A H_{\gamma_{n}}(\sigma)$ as $|\sigma|$. For the first case when $n=2$, a good way to choose $\gamma_{2}$ is to to have $\gamma_{2}: \mathbb{I}^{2} \rightarrow \Delta^{2}$ send the boundary of $\mathbb{I}^{2}$ to that of $\Delta^{2}$. In any way this is done, we would have that $d\left|\sigma_{2}\right|=\left|d \sigma_{2}\right|=0$, since the one-skeleton of $X$ is has been identified to a point. Looking forward, it is much better to choose $\gamma_{2}$ to be a "degree one" map $\mathbb{I}^{2} \rightarrow \Delta^{2}$ which when we consider the adjoint $\hat{\gamma}_{2}: \mathbb{I} \rightarrow \operatorname{Map}\left(\mathbb{I}, \Delta^{2}\right)$ interpolates between the direct path from vertex 0 to vertex 2 of $\Delta^{2}$ along the edge between them and the "long" path from 0 to 2 which first traverses the $0-1$-edge and then the 1-2 edge. When composed with the characteristic map into 
$X$ these edge paths will yield constant loops, but the choice of the paths in between is important See Fig. 2 for one choice of $\gamma_{2}$.

At the next stage, building on some such choice of degree one $\gamma_{2}$, we can define a $\gamma_{3}$ such that $d\left|\sigma_{3}\right|=\left|d \sigma_{3}\right|$. There are four faces of $\mathbb{I}^{2}$ and four faces of $\Delta^{3}$, and this equality identifies those faces. For example, one face of $\mathbb{I}^{2}$ will be mapped by $\hat{\gamma}_{3}: \mathbb{I}^{2} \rightarrow \operatorname{Map}\left(\mathbb{I}, \Delta^{3}\right.$ ) to paths from the 0 vertex to the vertex 3 (reminder: such a path in $\hat{X}$ will project to a loop in $X$ ) which first go to the vertex 1 directly along the $0-1$ edge and then go to 3 along paths compatible with the choice made of $\gamma_{2}$. On another face of $\mathbb{I}^{2}$, paths go only along the 0-2-3 face of $\Delta^{3}$, again compatibly with $\gamma_{2}$, and so on.

At $n=4$ the construction there is a surprise. Assume $\gamma_{3}$ has been defined, and start defining $\hat{\gamma_{4}}: \mathbb{I}^{3} \rightarrow \Delta^{4}$ by setting its restriction to various faces as before, for example sending one face of $\mathbb{I}^{3}$ to paths on the 0-2-3-4 face of $\Delta^{4}$. But, there are six faces of $\mathbb{I}^{3}$ and only five faces of $\Delta^{4}$ ! What is the natural last term? When one does the geometry carefully, one see that on the last face of $\mathbb{I}^{3}$ should map to paths from 0 to 4 which first go along the $0-1-2$ face and then along the 2-3-4 face. These two faces appear in the standard definition of the coproduct on simplicial chains dual to cup product. Moreover, such composites are given by the product in $C_{*}^{\square}(\Omega X)$. That is, we can construct $\gamma_{4}$ such that

$$
d\left|\sigma_{4}\right|=\left|d \sigma_{4}\right|+\left|\alpha_{2}\right| *\left|\beta_{2}\right|
$$

where the coproduct of $\sigma_{4}$ is $\alpha_{2} \otimes \beta_{2}$ plus terms in bidegrees $(1,3),(0,4)$, etc. (These other terms in the coproduct yield trivial chains with the 1-skeleton of $X$ collapsed.)

In general, let us denote products of Adams-Hilton chains by $|\sigma| *|\tau|=|\sigma| \tau \mid$. Let $\operatorname{Cobar}\left(C_{*}^{\Delta}(X)\right)$ denote the sub-algebra of $C_{*}^{\square}(\Omega X)$ generated by the Adams-Hilton chains (in positive degrees).

Theorem 1.3 (Adams-Hilton) There are degree-one choices for the maps $\gamma_{n}$ such that the boundary on $\operatorname{Cobar}\left(C_{*}^{\Delta}(X)\right)$ is the cofree extension of the map with

$$
d|\sigma|= \pm|d \sigma|+\sum_{\bar{\Delta} \sigma=\sum \alpha_{i} \otimes \beta_{i}} \pm\left|\alpha_{i}\right| \beta_{i} \mid .
$$

Here $\bar{\Delta}$ denotes the reduced cup coproduct including terms of only positive bidgrees.

The inclusion of any such Cobar $\left(C_{*}^{\Delta}(X)\right)$ in $C_{*}^{\square}(\Omega X)$ is a quasi-ismorphism of differential graded associative algebras.

Exercise 2 Try to write down $\gamma_{n}$ for $n \leq 4$ as an explicit piecewise-linear map.

Exercise 3 The cobar construction is defined for any differential graded coalgebra. Compute it for the coalgebra given by the homology of $\mathcal{C} P^{\infty}$.

Exercise 4 Deduce the James theorem from the Adams-Hilton theorem.

The algebraic cobar construction (often denoted $\Omega$ but not at the moment because of potential confusion) has become part of the standard toolkit for algebraic topologists, 
and there are more algebraic approaches which can yield similar theorems. A more geometric approach to the topology of iterated loop spaces was extended by Milgram who studied $\Omega^{n} \Sigma^{n} X$ in [11] (see also [4]). But the geometry and formalism of PROPs and operads, in particular the elegance of the little disks construction of Boardman and Vogt [3], became more popular than this intricate geometry. Perhaps there could be something gained by revisiting these ideas.

\subsection{Classifying spaces and the bar construction}

We will be more brief about the bar construction, whose topology is better known. The topological bar construction provides a model for the classifying space $B G$, which when $G$ is discrete is just an Eilenberg-MacClane space $K(G, 1)$.

Topologists are often ambiguous and refer to any quotient of a contractible space $E G$ with free $G$-action as the classifying space $B G$. We resolve this issue by only saying that such a space is homotopy equivalent (rather than equal to) $B G$, which we choose unambiguously to be the realization of the bar construction, which we partially define below.

Example $1.4 \bullet B \mathbb{Z} \simeq S^{1}$.

- $B \mathbb{Z} / 2 \simeq \mathbb{R} P^{\infty}$.

- $B \mathbb{Z} / n \simeq S^{\infty} /(\mathbb{Z} / n)$, called an infinite Lens space.

- If $G=\pi_{1}(S)$ where $S$ is a surface of positive genus, then $B G \simeq S$.

For simplicity, we present the "unreduced" bar construction, which lacks identifications coming from the identity element of $G$.

Theorem 1.5 If $G$ is discrete, then BG is homotopy equivalent to a simplicial complex whose $n$-simplices are in one-to-one correspondence with $n$-tuples of elements of $G$, which we denote $\left|g_{1}\right| g_{2}|\cdots| g_{n} \mid$. The $(n+1)$ faces of an $n$-simplex are given by

$$
d_{i}\left(\left|g_{1}\right| \cdots\left|g_{n}\right|\right)= \begin{cases}\left|g_{2}\right| \cdots\left|g_{n}\right| & i=0 \\ \left|g_{1}\right| \cdots\left|g_{i} g_{i+1}\right| \cdots\left|g_{n}\right| & 0<i<n \\ \left|g_{1}\right| \cdots\left|g_{n-1}\right| & i=n .\end{cases}
$$

To prove this, one constructs $E G$ in a similar fashion.

Corollary 1.6 The homology of BG is given by the homology of the algebraic bar construction applied to the group ring $\mathbf{k}[G]$, an associative algebra.

Exercise 5 Do the simple unraveling of definitions to check that this corollary follows.

We obtain a better model if we quotient by identifying each $n$-simplex of the form $\left|g_{1}\right| \cdots|e| g_{i+1}|\cdots| g_{n} \mid$ with the $(n-1)$-simplex $\left|g_{1}\right| \cdots\left|g_{i-1}\right| g_{i+1}|\cdots| g_{n} \mid$ through the appropriate standard projection of $\Delta^{n} \rightarrow \Delta^{n-1}$. The following exercise is a must for any topology student.

Exercise 6 Show that this reduced construction for $\mathbb{Z} / 2$ is homeomorphic to $\mathbb{R} P^{\infty}$. 
Thus $\mathbb{R} P^{\infty}$ has $\mathbb{Z} / 2$ as its DNA, so to speak. Theorem 1.5 is true in greater generality in particular when $G$ has a topology (with some mild assumptions) which gets incorporated in the topology on $B G$, or when $G$ is just a monoid. Indeed, this construction is a special case of the nerve of a category.

\subsection{Relating the bar and cobar constructions}

Because $B G=E G / G$ its homotopy type essentially defined through the fiber sequence

$$
G \subset E G \rightarrow B G
$$

Let $P X$ denote the path space on $X$, which is contractible, and let $e v$ denote the map which sends a path $\gamma$ to $\gamma(1) \in X$. Then the sequence

$$
\Omega X \rightarrow P X \stackrel{e v}{\rightarrow} X
$$

is a fibration. Consider as well the map $P E G \rightarrow B G$ defined by evaluation composed with the quotient. This map is equivalent to both the projection $E G \rightarrow B G$ and the evaluation $P B G \rightarrow B G$, which are thus equivalent to each other. We deduce that their fibers are equivalent, so that $\Omega B G \simeq G$. Similarly, if $X$ is connected then $B \Omega X \simeq X$ (the content of this statement depends on the definition of classifying space for $\Omega X$; some say its classifying space is $X$ by definition).

These homotopy equivalences are reflected in the following algebra, which is now viewed as a consequence of Koszul duality of the associative operad. Recall that the cobar construction on the chains of $X$ - a differential graded coalgebra-was defined in terms of a free associative algebra (and indeed computed the homology of $\Omega X$ as an algebra) with a differential comprised of both the internal differential and one defined using comultiplication. We can view the bar construction as based on the (co)free coassociative coalgebra generated by $\mathbf{k}[G]$, with the coproduct defined by breaking bar expressions in two and differential defined using the product of $G$. More generally, we may start with a differential graded associative algebra and define the bar construction as the total complex of the cofree coassociative coalgebra as a bicomplex, using both the internal differential and a differential defined by "removing bars and multiplying."

Theorem 1.7 The bar construction B and the cobar construction $\Omega$ define an adjoint pair of functors between differential graded associative algebras DGAA and differential graded associative coalgebras DGAC.

$$
\text { DGAC } \underset{B}{\stackrel{\Omega}{\rightleftarrows}} \mathrm{DGAA}
$$

Moreover, there are natural transformations $\Omega B A \rightarrow A$ and $B \Omega C \rightarrow C$ which are quasi-isomorphisms if $A$ is positively graded and if $C$ is 1-connected respectively.

This theorem was announced by Moore [13], so it has historically been referred to as Moore duality. In topology, this equivalence reflects the bijection between homoto- 
py classes of monoid maps from some $M$ to $\Omega X$ and homotopy classes of maps from $B M$ to $X$.

Not only is it the first example of adjoint functors giving equivalences between categories of algebras and coalgebras over an operad and its Koszul dual, but it played a central role in Priddy's definition of Koszul quadratic algebras [14]. A graded augmented algebra $A$ can be given a zero differential. Over a field $\mathbf{k}$ and with finiteness degree-wise, the homology of the bar complex of $A$ is the linear dual of $\operatorname{Ext}_{A}(\mathbf{k}, \mathbf{k})$, compatible with their coalgebra and algebra structures. (In the case of $A=\mathbf{k}[G]$, this is reflected by Corollary 1.6 and the fact that the cohomology of $B G$ is coincides with $\operatorname{Ext}_{\mathbf{k}[G]}(\mathbf{k}, \mathbf{k})$.) If $A$ is a Koszul algebra (given zero differential), then we can replace the bar complex with a much smaller resolution, which leads to an explicit presentation of this Ext-algebra. Moreover, the theory applies to this Ext-algebra as well and replaces the cumbersome quasi-isomophism of $A \simeq \Omega B A$ with an isomorphism $A \cong \operatorname{Ext}_{\operatorname{Ext}_{A}(\mathbf{k}, \mathbf{k})}(\mathbf{k}, \mathbf{k})$. In general, there is a spectral sequence relating the two.

\section{Other ideas in the air}

Following up on his thesis, Serre along with Cartan considered the rational homotopy groups of a simply connected space. When shifted down, as best done by considering the homotopy groups of $\Omega X$, those groups form a graded Lie algebra. Typically the Hurewicz homomorphism from homotopy to homology captures little information. But rationally for loop spaces, this map gives a clear picture. Building on calculations of Cartan and Serre [5], Milnor and Moore in [12] prove the following.

Theorem 2.1 If $X$ is simply connected, the Hurewicz map $\pi_{*}(\Omega X) \otimes \mathbb{Q} \rightarrow$ $H_{*}(\Omega X ; \mathbb{Q})$ is an injection, mapping the rational homotopy Lie algebra of $X$ to the primitives in the Hopf algebra $H_{*}(\Omega X ; \mathbb{Q})$.

Another influential idea at that time was Eckmann-Hilton "Duality," which draws attention to parallel structures in cohomology and homotopy. See the table below.

\begin{tabular}{ll}
\hline Cohomology & Homotopy \\
\hline L.E.S of a cofibration $A \hookrightarrow X \rightarrow X / A$ & L.E.S of a fibration $F \rightarrow E \rightarrow B$ \\
Spheres and Moore spaces & Eilenberg-MacClane spaces \\
Suspension/desuspension & Loop space/classifying space \\
CW structures & Postnikov tower \\
Graded commutative ring structure & Graded Lie algebra structure \\
Co- $H$-space (comonoid) & $H$-space (monoid) \\
Pushout square/homotopy colimit & Pull-back square/homotopy limit \\
Steenrod algebra & Stable homotopy groups of spheres \\
Leray-Serre spectral sequence & Blakers-Massey theorems \\
\hline
\end{tabular}

This duality is more of a philosophy than a theory. There are no theorems of the form "Given a true statement about homotopy groups, there is a true statement about cohomology groups obtained by..." or "Given a space $X$ there is a dual space $\hat{X}$ whose cohomology groups are the homotopy groups of $X$ and..." Nonetheless, the duality can point to interesting directions of study. For example, looking at our table 
one notices a significant difference between CW structures, which are not canonical in any sense, and the Postnikov tower, which is. This leads to finding the homology decomposition of a space (see Chapter 4.H of [9] for a basic treatment).

\section{Quillen functors and rational homotopy theory}

Quillen, influenced by Kan, took the step in [15] of proving theorems not about homotopy groups but about all of homotopy theory. He must have taken Theorems 2.1 and 1.3 as an important starting point. Indeed, if the rational homology of the cobar construction computes the homology of the loop space, and one is to then take primitives to get rational homotopy groups, why not take primitives first at the level of the cobar complex itself (see exercise below)? The great advantage is that in the cobar construction one is considering the free associative algebra, whose primitives are known to be the free Lie algebra, so one can just use the free Lie algebra functor as a starting point. Quillen was also aware of Chevalley-Eilenberg cohomology of Lie algebras [6], and probably knew of some cases in which applying this functor to the rational homotopy Lie algebra of a space recovered its cohomology (an easy case being wedges of spheres, whose rational homotopy Lie algebra is free). Once again, a refinement is needed, going from applying a functor at the level of algebras (in the previous case primitives, in the current case Lie algebra cohomology) to applying it at the level of chain complexes. Quillen's adaption of the Chevalley-Eilenberg construction now bears his name as well.

Quillen put these two constructions together in the following theorem.

Theorem 3.1 The Lie algebraic cobar construction $\Omega_{\mathcal{L i e}}$ and a commutative coalgebraic bar construction $B_{C}$ omm, which generalizes the Chevalley-Eilenberg construction, form an adjoint pair of functors

$$
\text { DGCC } \underset{B_{\text {Comm }}}{\stackrel{\Omega_{\mathcal{L i e}}}{\rightleftarrows}} \text { DGLA }
$$

Here DGCC are 1-connected differential graded cocommutative coalgebras and DGLA are connected differential graded Lie algebras. These functors preserve all notions relevant to homotopy theory (fibrations, cofibrations, weak equivalences).

Any simply-connected space $X$ has functorial models $C_{X}$ and $L_{X}$ in DGCC and DGLA, respectively, such that the homology of $C_{X}$ is the rational homology coalgebra of $X$ and the homology of $L_{X}$ is the rational homotopy Lie algebra of $X$.

In current language, we would say that $\Omega_{\mathcal{L} i e}$ and $B_{\mathcal{C} o m m}$ form a Quillen adjoint pair of functors on the model categories DGCC and DGLA, reflecting the Koszul duality of the operads $\mathcal{L}$ ie and Comm. This theorem gives a precise manifestation of EckmannHilton duality, through the fact that these functors preserve model structures along with the symmetries of the model structure axioms. What complicates [15] significantly is that there is, to this day, no simple way to construct a commutative cochain algebra of a space and thus easily land in this picture. Quillen has to walk for 40 days through the desert, producing a long chain of functors in order to produce $L_{X}$ and 
$C_{X}$. That difficulty led Sullivan to find a simple way to produce commutativity, not on chains but on cochains. Additionally, instead of using bar or cobar constructions Sullivan studied cofibrant replacements with some additional smallness property, the famous minimal models of [18].

Exercise 7 Check directly in some cases that the primitives of differential graded Hopf algebra form a split sub-complex, so that the primitives of the homology of $C$. is isomorphic to the homology of the complex obtained by taking the primitives of $C_{\bullet}$.

Exercise 8 Compute the Chevalley-Eilenberg cohomology of the graded Lie algebra with three generators $x, y, z$ in degree three with the only relation being $[x, y]=[y, z]$.

\section{Koszul duality and Hopf invariants}

We have recently found [17] that Koszul duality and Quillen functors allow one to give a definitive treatment of rational homotopy functionals through Hopf invariants. The basic idea can be seen as using the bar complex to understand a map $f: S^{n} \rightarrow X$ by first passing to $\Omega f: \Omega S^{n} \rightarrow \Omega X$ and then evaluating cohomology classes of $\Omega X$ on the image of the fundamental class of $\Omega S^{n}$. By Theorem 2.1, such invariants of rational homotopy classes of maps are complete.

We must pause to make a choice in notation. If one is studying the cohomology of $\Omega X$ using the cochains on $X$, one could either denote the construction you use by $\Omega$ to reflect topology or $B$ to denote a bar construction which is applied to an algebra (rather than a cobar construction applied to a coalgebra). Despite the fact that the bar construction here is really an algebraic dual to the cobar construction, lacking the first and last terms in the differential, we follow convention and let $B C^{*}(X)$ denote this "modified" bar construction. We let $H_{B}^{*}(X)$ denote the homology of $B C^{*}(X)$. Define the weight of a generator of a bar complex to be the number of elements appearing.

Lemma 4.1 $H_{B}^{n-1}\left(S^{n}\right)$ is rank one, generated by an element of weight one, namely $|\iota|$ where $\iota$ is a cocycle which represents the generator of $H^{n}\left(S^{n}\right)$.

Exercise 9 Prove this. Hint: you'll need the Künneth theorem to put yourself in a position to do some "weight reduction," as we use below.

Definition 4.2 Let $\gamma \in B^{n-1}\left(C^{*} S^{n}\right)$ be a cocycle. Define $\tau(\gamma) \simeq \gamma$ to be a choice of weight one cocycle to which $\gamma$ is cohomologous.

Define $\int_{B\left(S^{n}\right)}$ to be the map from cocyles in $B^{n-1}\left(C^{*} S^{n}\right)$ to $\mathbb{Z}$ given by $\int_{B\left(S^{n}\right)} \gamma=$ $\int_{S^{n}} \tau(\gamma)$, where $\int_{S^{n}}$ denotes evaluation on the fundamental class of $S^{n}$.

Define $\eta(\gamma)$, the Hopf invariant associated to $\gamma$ by $\eta(\gamma)(f)=\int_{B\left(S^{n}\right)} f^{*} \gamma$.

The choice of Hopf cochain is not unique, but the corresponding Hopf invariants are. It is immediate that the Hopf invariants are functorial. Moreover, note that the definitions hold with any ring cofficients. Topologically we have the following interpretation.

Proposition $4.3 \eta(\gamma)(f)$ coincides with the evaluation of the cohomology class given by $\gamma$ in $H^{n-1}(\Omega X)$ on the image under $\Omega f$ of the fundamental class in $H_{n-1}\left(\Omega S^{n}\right)$. 


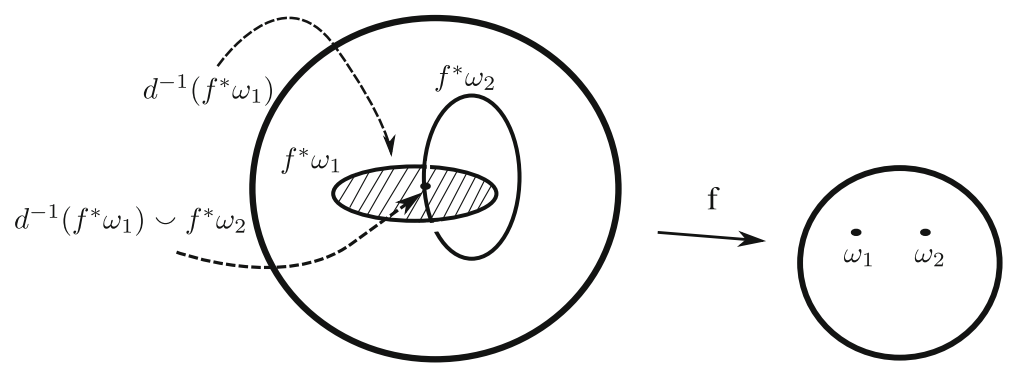

Fig. 3 Whitehead's integral, viewed through intersections of supports of cochains

\subsection{Examples}

Example 4.4 A cocycle of weight one in $B\left(C^{*} X\right)$ is just a closed cochain on $X$, which may be pulled back and immediately evaluated. Decomposable elements of weight one in $B(X)$ are null-homologous, consistent with the fact that products evaluate trivially on the Hurewicz homomorphism.

Example 4.5 Let $\omega_{1}$ and $\omega_{2}$ be generating 2-cocycles on $S^{2}$ and $f: S^{3} \rightarrow S^{2}$. Then $\gamma=-\left|\omega_{1}\right| \omega_{2} \mid$ is a cocycle in $B\left(C^{*} S^{2}\right)$ which $f$ pulls back to $-\left|f^{*} \omega_{1}\right| f^{*} \omega_{2} \mid$, a weight two cocycle of total degree two on $S^{3}$. Because $f^{*} \omega_{1}$ is closed and of degree two on $S^{3}$, it is exact. Let $d^{-1} f^{*} \omega_{1}$ be a choice of a cobounding cochain. Then

$$
d\left(\left|d^{-1} f^{*} \omega_{1}\right| f^{*} \omega_{2} \mid\right)=\left|f^{*} \omega_{1}\right| f^{*} \omega_{2}|+| d^{-1} f^{*} \omega_{1} \cup f^{*} \omega_{2} \mid
$$

Thus $f^{*} \gamma$ is homologous to $\left|d^{-1} f^{*} \omega_{1} \cup f^{*} \omega_{2}\right|$, and the corresponding Hopf invariant is $\int_{S^{3}} d^{-1} f^{*} \omega_{1} \cup f^{*} \omega_{2}$, which when choosing $\omega_{1}=\omega_{2}$ is the classical formula for Hopf invariant given by Whitehead [19]. It is a direct translation of the linking number definition of Hopf invariant into the language of cochains see Fig. 3. Understanding this formula from the point of view of the bar construction has, to our knowledge, only come over 50 years since all of these concepts were introduced.

Example 4.6 For an arbitrary $X$ and cochains $x_{i}, y_{i}$ and $\theta$ on $X$ with $d x_{i}=d y_{i}=0$ and $d \theta=\sum(-1)^{\left|x_{i}\right|} x_{i} \cup y_{i}$, the cochain $\gamma=\sum\left|x_{i}\right| y_{i}|+| \theta \mid \in B\left(C^{*} X\right)$ is closed. The possible formulae for the Hopf invariant are all of the form

$$
\int_{S^{n}}\left(f^{*} \theta-\sum\left((-1)^{\left|x_{i}\right|} t_{i} \cdot d^{-1} f^{*} x_{i} \cup f^{*} y_{i}+\left(1-t_{i}\right) \cdot f^{*} x_{i} \cup d^{-1} f^{*} y_{i}\right)\right),
$$

for some real numbers $t_{i}$. This generalizes formulae given in [7,8,18].

By choosing $t=\frac{1}{2}$ we see that reversing the order to consider $\sum\left|y_{i}\right| x_{i} \mid$ will yield the same Hopf invariant, up to sign. Thus $\sum\left|x_{i}\right| y_{i}|\mp| y_{i}\left|x_{i}\right|$ yields a zero Hopf invariant. There are many Hopf invariants of the classical bar construction which are zero, a defect remedied by using the Lie coalgebra cobar construction. 
Exercise 10 Suppose $x$ and $y$ are cochains supported on codimension two submanifolds $X$ and $Y$ of $W$ and $\theta$ satisfies $d \theta=x \cup y$ and is supported on a codimension three submanifold which cobounds $X \cap Y$. Draw pictures of how the Hopf invariant associated to $|x| y|\mp| \theta \mid$ evaluates some map $f: S^{3} \rightarrow X$. Moreover, draw pictures of what can happen in $S^{3} \times \mathbb{I}$ if one has a homotopy between $f$ and $g$. [Hint: Start with the picture in the figure, but then draw in the preimage of the support of $\theta$; then, think about what can happen with the preimage of $X \cap Y$ through a homotopy.]

One can do similar calculations in higher weight, and interpret them all when one chooses cochains supported on submanifolds in terms of linking behavior of the preimages of those submanifolds. See [17].

\subsection{The cokernel and kernel of the Hopf invariant map}

Our Hopf invariant construction defines a homomorphism $\eta: H_{*}\left(B\left(C^{*}(X ; \mathbb{Z})\right)\right) \rightarrow$ $\operatorname{Hom}\left(\pi_{*} X, \mathbb{Z}\right)$. It follows from Proposition 4.3 and Theorem 2.1 that this map is surjective when tensored with the rational numbers, and thus is full rank.

Problem 11 Compute the cokernel of $\eta$. By Adams' celebrated result [1], this cokernel is trivial for $X$ an odd sphere and for $S^{2}, S^{4}$ and $S^{8}$, and it is $\mathbb{Z} / 2$ for other even spheres.

The proofs in [17] show that one might be able to directly understand the relation of this cokernel to lack of commutativity of cup product. Though this cokernel is clearly a very subtle homotopy invariant, we do not see any applications of its calculation.

Also, $\eta$ has a very large kernel, explained from the operadic viewpoint as the fact that we are taking the wrong bar construction. The rational PL cochains on a simplicial set are commutative, so we should be taking a bar construction over the Koszul dual cooperad, namely the Lie cooperad, rather than associative cooperad. The homology of such a bar construction $B_{\mathcal{L i e}}$ is known as Harrison homology. Using a graphical model for the Lie cooperad developed in [16] which makes calculations possible, we prove the following.

Theorem 4.7 [17] There is a Hopf invariant map $\eta^{\text {Lie }}$ which factors the map $\eta$ such that $\eta^{\text {Lie }}: H_{B_{\mathcal{L i e}}^{*}}^{*}(X) \rightarrow \operatorname{Hom}\left(\pi_{*}(X), \mathbb{Q}\right)$ is an isomorphism of Lie coalgebras.

It is almost immediate that similar Hopf invariants can be used to concretely realize similar isomorphisms arising for Koszul pairs in general.

To summarize, in homology theory it has been helpful to have geometry attached not only to homology but cohomology. In particular, homology classes are often represented by compact submanifolds and cohomology classes are represented by either forms or proper submanifolds. The geometry of homotopy groups arising from their definition is almost too simple. Our work on Hopf invariants shows that the geometry of homotopy functionals is given by linking invariants, as perfectly governed by the Lie cooperad, completing the geometric understanding of these basic functors in the rational setting. We hope these ideas can be extended to the non-simply connected setting, and perhaps_-at least in part—in characteristic $p$. 
Acknowledgments I would like to thank my collaborator Ben Walter. This material is a union of standard material which either I taught him or he taught me along with new theorems which we have figured out together.

\section{References}

1. Adams, J.F.: On the non-existence of elements of Hopf invariant one. Ann. Math. (2) 72, 20-104 (1960) (MR0141119 (25 \#4530))

2. Adams, J.F., Hilton, P.J.: On the chain algebra of a loop space. Comment. Math. Helv. 30, 305-330 (1956) (MR0077929 (17,1119b))

3. Boardman, J.M., Vogt, R.M.: Homotopy invariant algebraic structures on topological spaces. In: Lecture Notes in Mathematics, vol. 347. Springer, Berlin (1973) (MR0420609 (54 \#8623a))

4. Carlsson, G., James Milgram, R.: Stable homotopy and iterated loop spaces. In: Handbook of algebraic topology, pp. 505-583. North-Holland, Amsterdam (1995) (MR1361898 (97j:55007))

5. Cartan, H., Serre, J.-P.: Espaces fibrés et groupes d'homotopie. II. Applications. C. R. Acad. Sci. Paris 234, 393-395 (1952) (MR0046046 (13,675b))

6. Chevalley, C., Eilenberg, S.: Cohomology theory of Lie groups and Lie algebras. Trans. Am. Math. Soc. 63, 85-124 (1948) (MR0024908 (9,567a))

7. Griffiths, P.A., Morgan, J.W.: Rational homotopy theory and differential forms. In: Progress in Mathematics, vol. 16. Birkhäuser, Boston (1981) (MR641551 (82m:55014))

8. Haefliger, A.: Whitehead products and differential forms, Differential topology, foliations and Gelfand-Fuks cohomology. In: Lecture Notes in Mathematics, vol. 652, pp. 13-24. Springer, Berlin (1978) (MR505648 (80a:55012))

9. Hatcher, A.: Algebraic topology. Cambridge University Press, Cambridge (2002) (MR1867354 (2002k:55001))

10. James, I.M.: Reduced product spaces. Ann. Math. (2) 62, 170-197 (1955) (MR0073181 (17,396b))

11. Milgram, R.J.: Iterated loop spaces. Ann. Math. (2) 84, 386-403 (1966) (MR0206951 (34 \#6767))

12. Milnor, J.W., Moore, J.C.: On the structure of Hopf algebras. Ann. Math. (2) 81, 211-264 (1965) (MR0174052 (30 \#4259))

13. Moore, J.C.: Differential homological algebra, Actes du Congrès International des Mathématiciens (Nice, 1970), Tome 1, pp. 335-339. Gauthier-Villars, Paris (1971) (MR0436178 (55 \#9128))

14. Priddy, S.B.: Koszul resolutions. Trans. Am. Math. Soc. 152, 39-60 (1970) (MR0265437 (42 \#346))

15. Quillen, D.: Rational homotopy theory. Ann. Math. (2) 90, 205-295 (1969) (MR0258031 (41 \#2678))

16. Sinha, D.P., Walter, B.: Lie coalgebras and rational homotopy theory, I, math.AT/0610437

17. Sinha, D.P.: Lie coalgebras and rational homotopy theory, II: Hopf invariants., arXiv:0809.5084

18. Sullivan, D.: Infinitesimal computations in topology. Inst. Hautes Études Sci. Publ. Math. 47, 269-331 (1978) (MR0646078 (58 \#31119))

19. Whitehead, J.H.C.: An expression of Hopf's invariant as an integral. Proc. Nat. Acad. Sci. USA 33, 117-123 (1947) (MR0020255 (8,525h)) 